\title{
Deletion of Alu Sequences in the Fifth c-sis Intron in Individuals with Meningiomas
}

\author{
Mary Smidt, * Ilan Kirsch, ${ }^{*}$ and Lee Ratner* \\ ${ }^{*}$ Departments of Medicine and Molecular Microbiology, Washington University, St. Louis, Missouri 63110; and ${ }^{\ddagger}$ Naval Medical \\ Oncology Branch, National Cancer Institute, National Institutes of Health, Bethesda, Maryland 20892
}

\begin{abstract}
An abnormality in the c-sis protooncogene was identified in leukocyte DNA from members of a family predisposed to the development of meningioma, and was found to be associated with the development of the tumor in those individuals. Molecular analysis of this abnormality demonstrated a deletion within the fifth intron of the c-sis gene. The normal c-sis gene has an Alu sequence in this region which includes two perfect 130 nucleotide repeated sequences separated by $5 \mathrm{bp}$. The deleted c-sis allele is missing precisely one copy of the $130 \mathrm{bp}$ repeat and the intervening $5 \mathrm{bp}$. An identical deletion was also found in DNA from 1 of 13 sporadic meningiomas. (J. Clin. Invest. 1990. 86:1151-1157.) Key words: PDGF • recombination • oncogene $\bullet$ PCR • sequence
\end{abstract}

\section{Introduction}

The c-sis protooncogene is located on human chromosome 22 (1). It encodes one of two polypeptides comprising platelet-derived growth factor (2). The c-sis gene is expressed in endothelial cells (3), activated monocytes (4), smooth muscle cells lining developing vasculature (5), astrocytes in the developing central nervous system (6), and tumors of fibroblastoid or glial origin (7). Transduction of this gene to produce the simian sarcoma virus conferred transforming properties on this retrovirus (8). This virus is capable of causing sarcomas and meningiomas in monkeys (9).

Human meningiomas have been associated frequently with complete or partial deletions of chromosome 22 (10). Homozygosity or hemizygosity of genes on chromosome 22 are found in $43 \%$ of meningiomas (11). Abnormalities in the region of the c-sis gene are found more frequently than those of other loci on this chromosome.

Individuals within a family predisposed to the development of meningioma have been described (12). An abnormality of the c-sis gene was closely associated with the development of the tumor. To elucidate the molecular basis of meningioma development in these individuals, we have cloned, mapped, and sequenced the abnormal c-sis allele from one such individual.

\section{Methods}

DNA sources. DNA from members of this family and a normal blood donor (L.R.) were obtained from fresh leukocytes or Epstein-Barr virus

Address reprint requests to Dr. Lee Ratner, Box 8125, 660 South Euclid, Washington University, St. Louis, MO 63110.

Received for publication 11 December 1989 and in revised form 20 March 1990.

J. Clin. Invest.

(c) The American Society for Clinical Investigation, Inc.

$0021-9738 / 90 / 10 / 1151 / 07 \$ 2.00$

Volume 86, October 1990, 1151-1157 immortalized lymphocytes. DNA was extracted (13) from sporadic meningiomas, the $\mathrm{H} 9$ human lymphoid cell line, the HOS human osteogenic sarcoma cell line, the A172 human glioblastoma cell line, and the SKES human sarcoma cell line using autoclaved reagents and utensils. Phage clone lambda SIS-A (14) includes exons 1-4 of the normal human c-sis gene (Fig. 3). Plasmid clones pL33-M, pL33-5, and $\mathrm{pB} 3 \mathrm{~L}$ include three contiguous Bam HI fragments from the normal human c-sis gene (2), whereas plasmid cDNA clone pSM-1 is from a human T-lymphotropic virus type 1 immortalized cell line (15) (Fig. 3).

Cloning and sequence analysis. A genomic library was prepared according to published procedures (13) using lambda EMBL3 (Promega Biotech., Madison, WI) and Mbo I partially digested DNA from lymphocyte DNA of patient II.8 (Fig. 1). The library was screened with the 1.8-kb Bam HI fragment of pL33-5 (Fig. 3), and six positive clones were obtained. The 3.8-kb Sac I fragment from one of these clones was subcloned into lambda ZAP (Stratagene Inc., La Jolla, CA) and excised according to the manufacturer's protocol in a Bluescript vector, designated pMD. A portion of $\mathrm{pL} 33-\mathrm{M}$ and $\mathrm{pMD}$ were sequenced on both strands using plasmid DNA by the partial chemical cleavage method (16; nucleotides 50-250 and 580-880; Fig. 4) and the dideoxynucleotide chain termination method (17; nucleotides 1-1200; Fig. 4) using Sequenase (United States Biochemical Corp., Cleveland, OH).

DNA blotting and hybridization. Digests of DNA were analyzed by Southern blot hybridization using probes labeled by the hexamer priming method (18).

Polymerase chain amplification reactions. Polymerase chain amplification reactions (PCR) ${ }^{1}$ were performed in $10 \mu \mathrm{l}$ with $150-500 \mathrm{ng}$ of cloned DNA, $200 \mu \mathrm{M}$ each of dNTP, $5 \mathrm{mM}$ sodium acetate, $10 \mathrm{mM}$ Tris- $\mathrm{Cl}, \mathrm{pH} 7.0,1.5 \mathrm{mM}$ magnesium chloride, and $1.0 \mu \mathrm{M}$ of each of the primers: $\mathrm{L}=$ TGGTGACCTTGTGCTTGCCTGGGCTTGTAT (239 nucleotides 5 ' to those shown in Fig. 4) and $P=$ CCGTCCGAATGGTCACCCGAGTTTGGGGCG (nucleotides 602-631 in exon 6 [2]) at $94^{\circ} \mathrm{C}$ for $3 \mathrm{~min}, 61^{\circ} \mathrm{C}$ for $3 \mathrm{~min}$, and $72^{\circ} \mathrm{C}$ for $10 \mathrm{~min}$ for 40 cycles. PCR products were analyzed on a $0.8 \%$ agarose gel which was stained with ethidium bromide.

\section{Results}

Restriction fragment analysis of a c-sis polymorphism. The pedigree of the family with predisposition to meningioma is shown in Fig. 1. Four individuals (I.1, II.2, II.3, and II.8) developed meningiomas at a median age $\sim 10$ years younger than that found for meningioma patients in the general population (12). Other tumors were noted in two individuals (II.4, ovarian carcinoma and rhabdomyosarcoma; and III.22, breast carcinoma). A Robertsonian translocation $\mathrm{t}(14 ; 22)$ (14qter $\rightarrow$ cen $\rightarrow$ 22qter) was noted in individuals II. 2 , II.8, III. 11 , III.12, III.13, and III.22, and presumed to be present in patient II.3. Pst I digestion of lymphocyte DNA from patients II.2, II.8 (MD), and III.21, and hybridization with the 1.8-kb Bam HI fragment of pL33-5 (Fig. 3) revealed fragments of 2.2, 3.3, and $3.4 \mathrm{~kb}$ (Figs. $2 a$ and 3; [12]). In contrast, Pst I digested DNA from individuals II.6, II.7, II.9, III.1 1, III.12, III.13, and III.22,

1. Abbreviations used in this paper: $\mathrm{PCR}$, polymerase chain amplification reaction. 

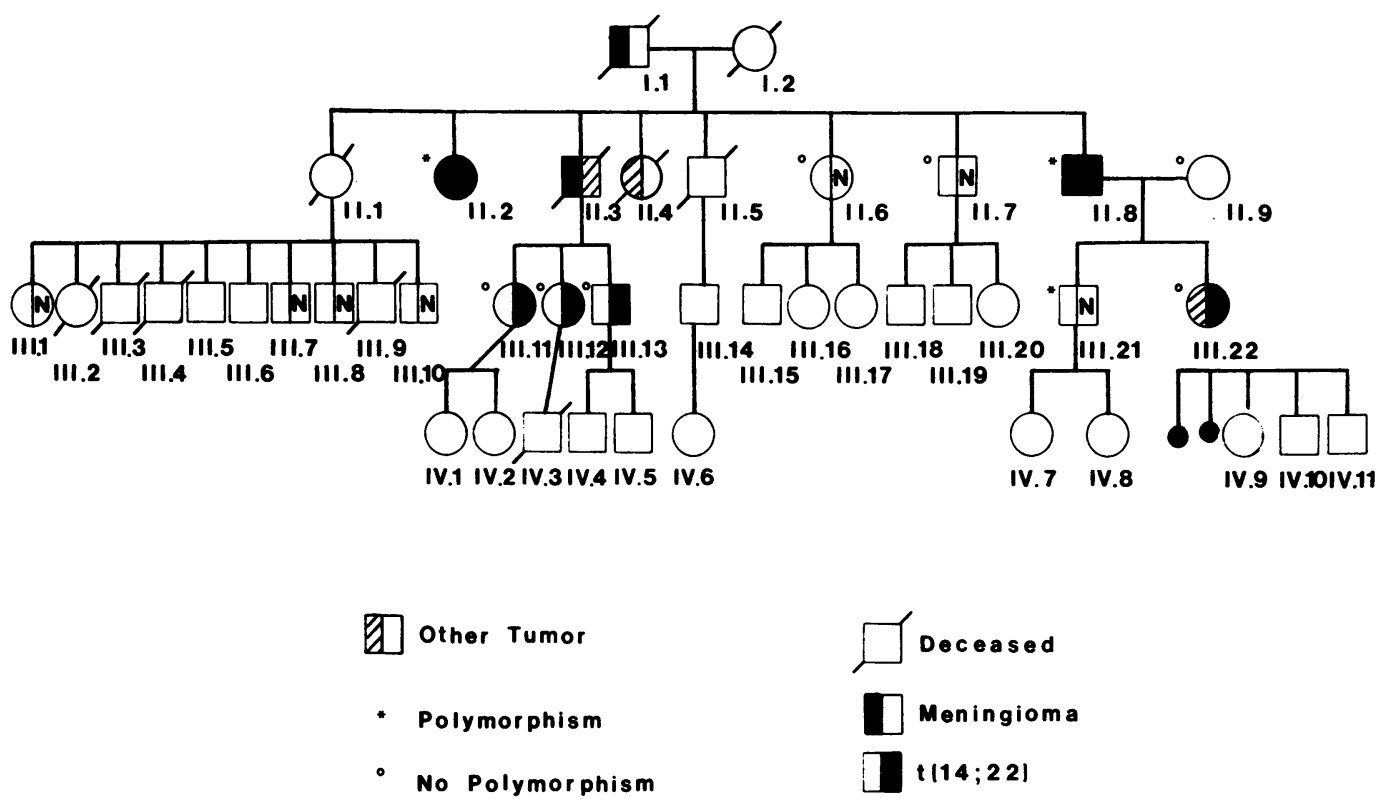

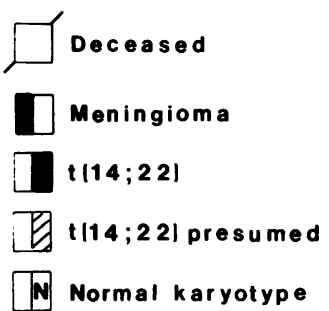

Figure 1. Polymorphism in the c-sis gene associated with development of meningioma. The pedigree for the family predisposed to meningioma development is shown. Individuals with meningioma or other tumors, demonstrated or presumed Robertsonian translocation, or normal karyotype, c-sis polymorphism, or deceased are identified. The small filled circles indicate spontaneous abortions. or 24 other unaffected and unrelated individuals revealed hybridizing fragments of 2.2 and $3.4 \mathrm{~kb}$ only (12; data not shown). Hybridization with a cDNA clone demonstrated no other alterations in the c-sis gene among these individuals (data not shown).

Digestion of control DNAs (A172, HOS, and L.R. in Fig. 2 b) with Bgl II demonstrated a doublet for $6.0-\mathrm{kb}$ fragments hybridizing with a cDNA probe, whereas digestion of control
DNAs (L.R., SKES, and H9 in Fig. 2 c) with Sac I demonstrated a doublet for 3.8-kb hybridizing fragments. In contrast, digestion of DNA from individual II.8 with Bgl II or Sac I demonstrated hybridizing fragments with a slightly greater electrophoretic mobility than those generated from control DNAs. This suggests that one fragment of each doublet is $\sim 0.1 \mathrm{~kb}$ smaller than those present in DNA from unaffected individuals (Fig. 2, $b$ and $c$ ). However, Bam HI digestion prod-

\section{a) Pst I}

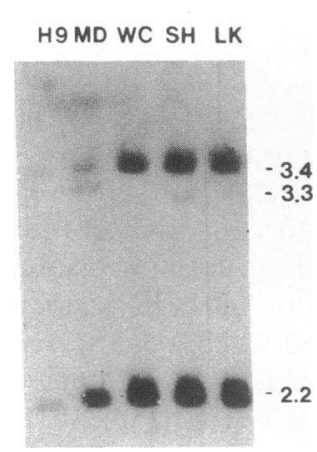

b) $\mathrm{Bgl} \mathrm{II}$
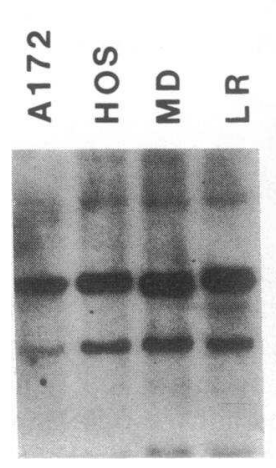

c) Sac I

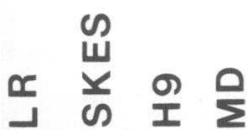

d) Pst I

Figure 2. Restriction enzyme analysis of the c-sis polymorphism. (a) Leukocyte DNAs from patient II.8 (M.D.) and neurofibromatosis type 1 patients (W.C., S.H., L.K.) and DNA from a T lymphoid cell line (H9) were digested with Pst I and analyzed by Southern blot hybridization with the 1.8-kb Bam HI pL33-5 probe (Fig. 3). DNA from patient M.D., an unrelated individual (L.R.), or cell lines (A172, HOS, SKES) were digested with either (b) Bgl II or (c) Sac I, and hybridized with the Sac II-Sac I probe of cDNA clone pSM1 which includes sequences from exons 2-7 (Fig. 3) (2). (d) DNAs from meningioma samples 1 and 16-19 and from H9 lymphoid cells are digested with Pst I and hybridized with the 1.8-kb Bam HI pL33-5 probe. The faint band for 2.6-kb DNA fragments represents weak cross-hybridization to another cellular sequence. This band is presented in the lane for tumor 1, but is less intense than for other samples because less DNA was loaded onto the lane of the gel. Size markers (in kilobases) are indicated to the right of each autoradiogram, and the positions of each fragment within the c-sis gene are shown in Fig. 3. 


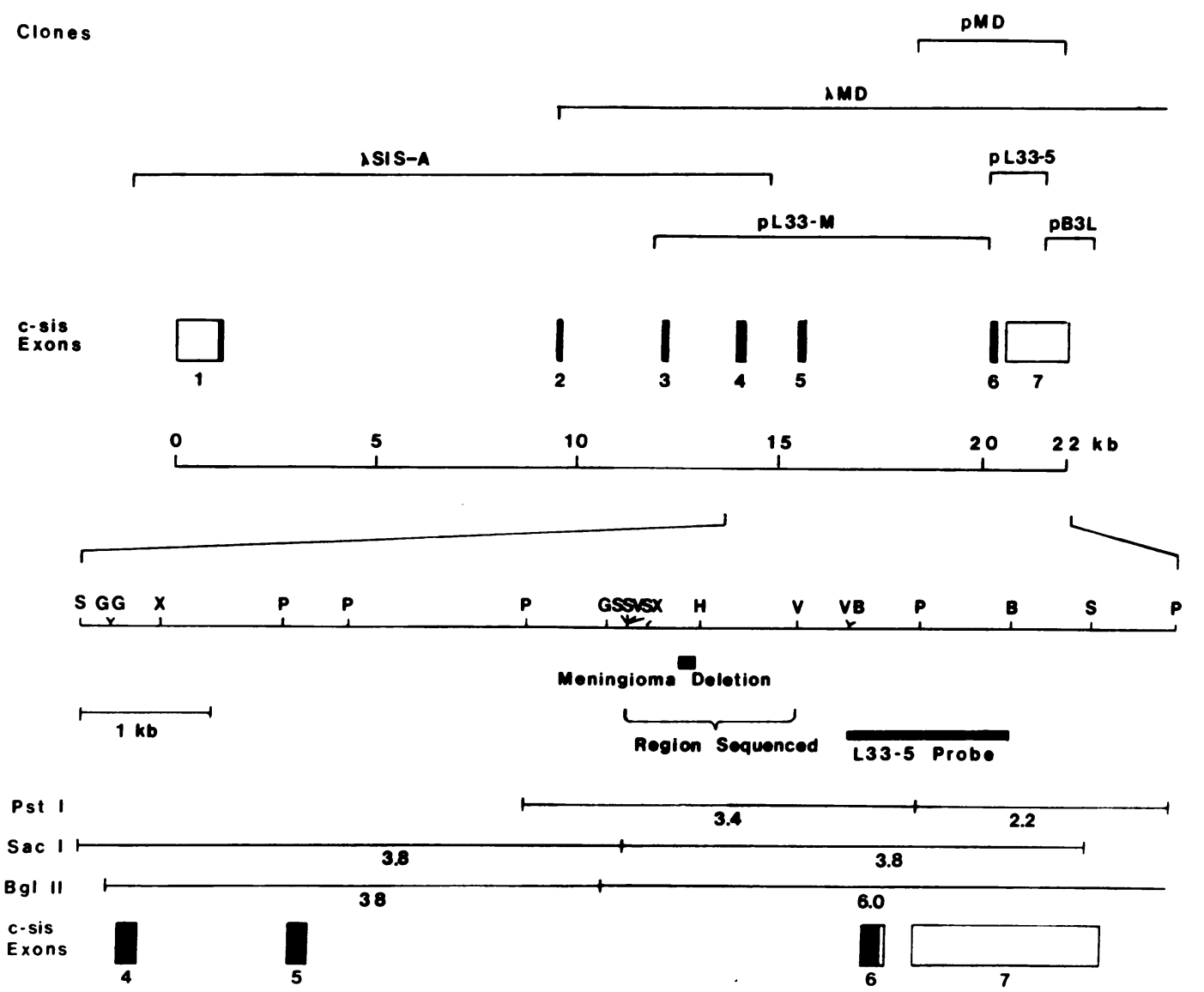

Figure 3. Characterization of a deleted c-sis allele. A restriction enzyme map of the c-sis gene shows the location of the deletion in the individual with meningioma. Boxes 1-7 indicate the positions of c-sis exons. Shaded portions of the boxes indicate the open reading frame for the B polypeptide chain of PDGF. A scale is shown below beginning at the RNA initiation site and ending at the RNA termination site. The bottom half of the figure is a magnified section of the $3^{\prime}$ portion of the gene. Relevant restriction enzyme sites and molecular clones used in the analyses are also shown. The site of the deletion in the patient with meningioma is indicated, as is the region sequenced (Fig. 4), and the L33-5 DNA fragment used as a hybridization probe (Fig. 2, $a$ and $d$ ). The hybridizing DNA fragments from the nondeleted allele are

indicated in kilobases after digestions with Pst I (Fig. 2, $a$ and $d$ ), Bgl II (Fig. $2 b$ ), or Sac I (Fig. $2 c$ ). Abbreviations: $B$, Bam HI; $G$, Bgl II; $H$, Sph I; $P$, Pst I; $S$, Sac I; $V$, Pvu II; $X$, Xba I.

ucts hybridizing with the pL33-5 probe were identical with DNAs from affected and unaffected individuals (not shown). Thus, the structural abnormality in the c-sis gene can be attributed to a deletion of $\sim 0.1 \mathrm{~kb}$ within the fifth intron of the c-sis gene (Fig. 3).

Isolation and characterization of the deleted c-sis allele. From a genomic library constructed with DNA from patient MD, six lambda clones were recovered which hybridized with the pL33-5 probe. One of these clones lacked any intron 5 sequences. Each of the other five clones generated DNA fragments of 1.7 and $3.8 \mathrm{~kb}$ after digestion with Sac I and Bam HI and hybridization with the 8.0-kb Bam HI pL33-M probe (Fig. 3; data not shown). In contrast, clone pL33-M from a normal individual generated 1.8 - and $3.8-\mathrm{kb}$ bands under these conditions. Additional restriction enzyme digests revealed the 0.1$\mathrm{kb}$ deletion in lambda MD to be localized between the Pvu II and Sph I sites in the fifth intron of the c-sis gene (Fig. 3). Digests of clones lambda SIS-A, lambda MD, pL33-M, and pL33-5 with Pst I, Bam HI, or Pst I and Bgl II, and hybridization with the $1.8-\mathrm{kb}$ Bam HI fragment of pL33-5, the $8.0-\mathrm{kb}$ Bam HI fragment of pL33-M, or the 15-kb Eco R I-Xba I fragment of lambda SIS-A revealed no other differences between the cloned c-sis alleles from affected and unaffected patients (data not shown).

Nucleotide sequence analysis of the normal and deleted c-sis alleles. A 1,200-bp region of intron 5 from pL33-M and pMD was sequenced (Fig. 4). pL33-M contains an Alu se- quence between nucleotides 405 and 821 . This Alu sequence is unusual in that it has two copies of the monomer 1 sequence, one having 5 and the other 16 nucleotide differences among the 133-bp of the consensus sequence described by Kariya and colleagues (19). These are followed immediately by a sequence with 17 differences among $149 \mathrm{bp}$ of the consensus monomer 2 sequence. An adenylate-rich 13-bp sequence follows the Alu sequence. The Alu sequence is also flanked by imperfect repeats of a 15-bp sequence with one difference from the consensus sequence. Within the Alu sequence are two copies of a perfect 130 -bp repeat separated by five nucleotides.

The sequence from pMD is identical to that from pL33-M except for a 135-bp deletion which includes one copy of the 130 -bp repeat and the five nucleotides between the direct repeats found in the normal allele (Fig. 4).

Polymerase chain amplification analysis of the $c$-sis gene in members of the family predisposed to meningiomas and individuals with sporadic meningiomas. A polymerase chain amplification assay was developed using primers which flanked the Alu repeat sequences. A 2.1-kb PCR product was identified using the cloned allele, pMD, whereas a 2.2-kb PCR product was noted with DNA from the H9 lymphoid cell lines (Fig. 5 a). Only a 2.2-kb product was found after PCR with leukocyte DNA from family members (III.13, III.12, III.11, and II.7) and individual II.9 who lacked the c-sis abnormality, while 2.1and 2.2-kb PCR products were found with DNA from family members (III.21, II.8, and II.2) with the c-sis abnormality. 
Sac I GAGCTCAAAAGGCTCCCAGGAAATGGCTGG̈CTTGAGCTCOGCAGACACAGCTGTCCCTGCCAGGGTGGC̈CACCTGGAAATCCAGGAOGGAGAGAAGTGT 100

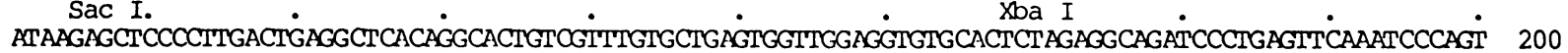

CICTCTGCTTCAGTTTCCTCATCTGTAAAGTGTGGGTAACAGCATCTGCOGGTAACAGCACCTGCOGTGANGGGAAATGÄGCATAGAACAGCACCAGGTA 300

CATGGTAAACACTAGCTGGGGGGTTTCTGGCCATTTCCATGTATTGTCTCCTAAOGCTAGGGGGCCAGGG̈CTGCTGTCTT'CTCTGCTTTAAAGATGTGGA 400 1--ALU FLANKING SEQUENCE-

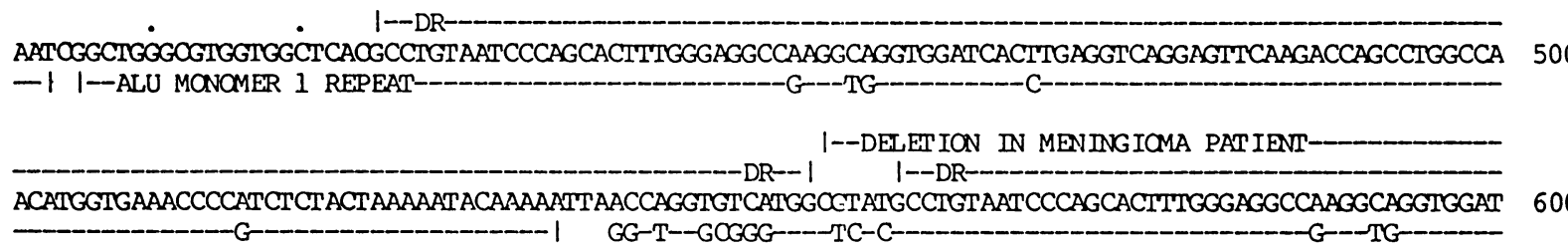

CACITGAGGTCAGGAGITCAAGACCAGCCTGGCCAACATGGTGAAACCCATCTCTACTAAAAATACAAAAATTAACCAGGIGTCATGGTGCATGCCTGT 700

AATCCCAGCTACTOGGGAGGCTGAGGCAGGAGAATCACTTGAACCTGOGAGGTGGFGGTIGCAGTGAGCIGAGATOGOGCCACTGCACTCCAACCTGGGC 800 ALU MONOMER 2 REPEAT-_-

AACAGAGTGíGCCTCOGTCITAAAAACAARTAAATAAAGATGCGGAAATÓGAGGCACAGGGGGGTTAAGTGACTTGCTCCAATGTCCACGATACGTGGAG 900

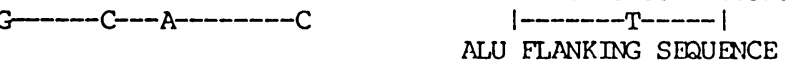

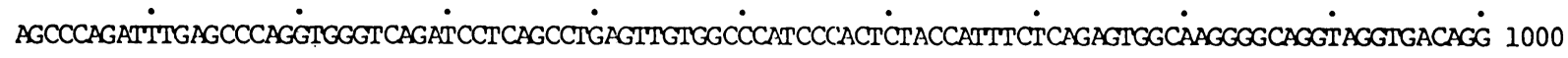

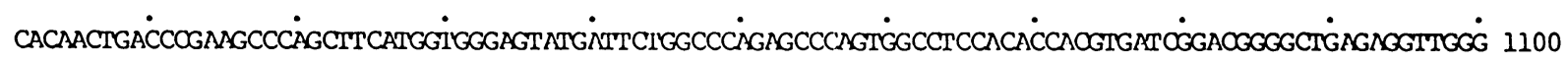
AGGAACCTCAATAGTAGGTCAAGCTAAGGAAATAAGAAAAAAAAGTAGTCGTAGCAGCTGCCATGTGCTGACCACTTCATACITGAGCTTTTTTITA 1200

Figure 4. Nucleotide sequences of the normal and deleted c-sis alleles. A 1,200-bp portion of the fifth intron of the c-sis gene from clone pL33$\mathrm{M}$ is shown. The corresponding region of the c-sis gene from clone pMD is identical except for the 135-bp deletion which is indicated. Positions of and differences with Alu repeat and flanking consensus sequences are shown, as well as the location of a precise direct repeat $(D R)$ sequence. Positions of relevant restriction enzyme sites are indicated. Every tenth nucleotide is indicated with a dot and the nucleotide position is indicated to the right of each line. These sequence data are available from EMBL/Gen Bank/DDBJ under accession number M38065.

The PCR assay was also utilized to screen DNA samples from brain tumor samples derived from unrelated individuals. All of the five nonmeningioma samples $(2,4,8,9$, and 13) produced only a 2.2-kb PCR product, while one (sample 1 ) of the eight meningioma tissues (samples $1,3,5,6,7,11,12$, and 14) produced PCR products of 2.1 and $2.2 \mathrm{~kb}$ (Fig. $5 \mathrm{~b}$ ).

To assess the structural abnormality in meningioma No. 1 , Southern blot hybridization was performed after Pst I digestion of fresh tissue (Fig. $2 d$ ). The presence of 3.4- and 3.3-kb bands demonstrates a polymorphism in this sample similar to that found in the members of the family predisposed to the development of meningioma. In contrast, Pst I digestion of DNAs from five other meningiomas (samples 6 and 16-19) reveal only the 3.4-kb band.

Differences in the relative intensities of bands from deleted and nondeleted alleles between the PCR and Southern blot analyses for patients II.8 (M.D.; Figs. 2 and $5 a$ ) and patient 1 (Figs. $2 d$ and $5 b$ ) are likely due to differences in efficiency of PCR for the two different DNA fragments.

The 2.1-kb PCR product from meningioma No. 1 was isolated and cloned into a plasmid. Sequence analysis of nucleotides $255-758$ of the fifth intron revealed a deletion identical to that found in pMD (Fig. 4).

\section{Discussion}

Meningiomas are common tumors accounting for $15-18 \%$ of all intracranial neoplasms (20). They have been found in $1.4 \%$ of autopsies (21). Though they are usually benign, they often require treatment because of compressive effects on adjacent brain tissue (22). Meningiomas may occur in a familial form associated with neurofibromatosis or without these typical clinical stigmata (23-25). The family described here is remarkable for the development of meningioma in four individuals over two generations. Though a Robertsonian translocation involving chromosome 22 is noted in several members of this family, this translocation was not as tightly associated with meningioma development as the abnormality in the c-sis gene. Such a translocation is an incidental finding in $\sim 1 \%$ of normal individuals (26). 

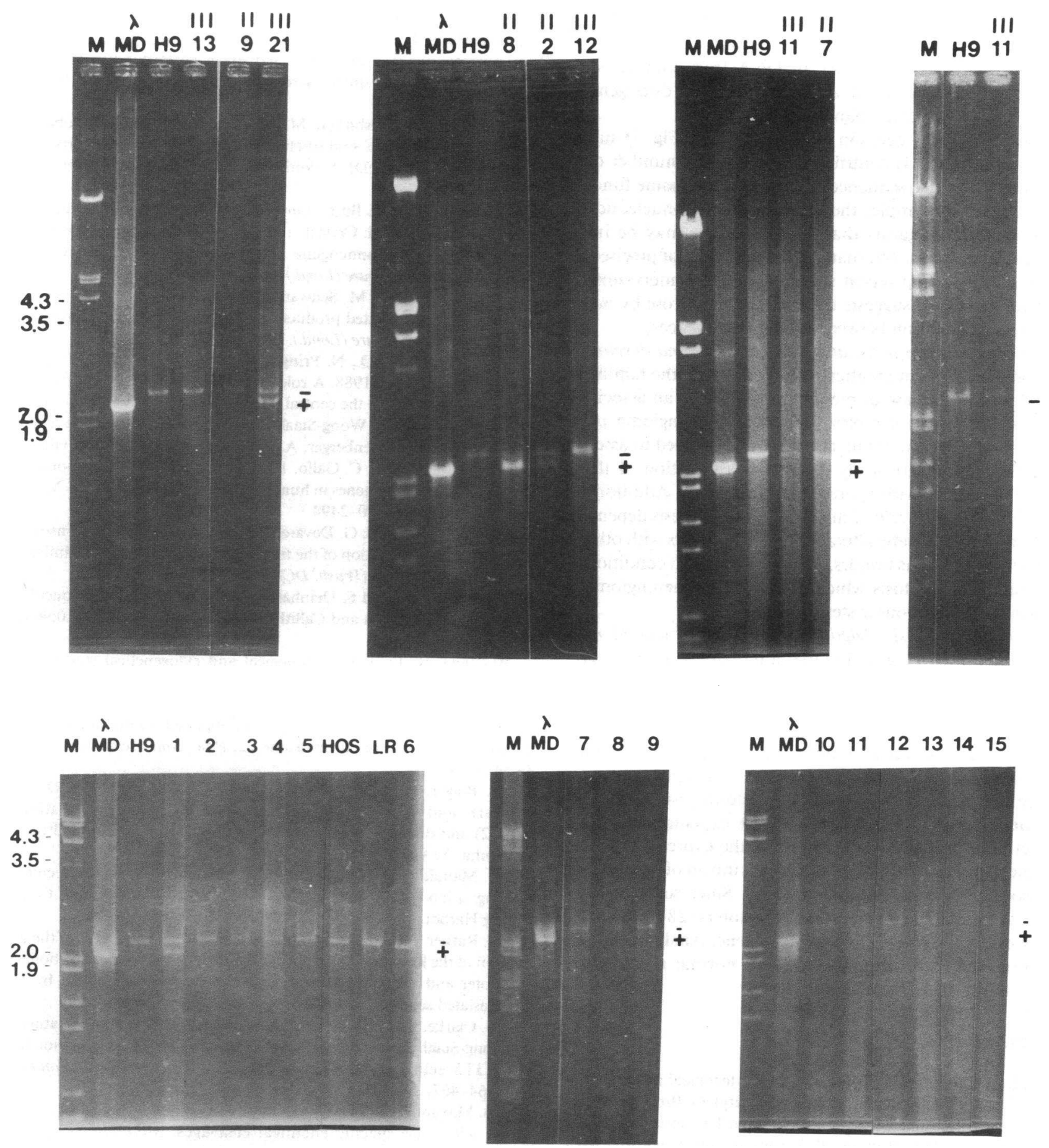

Figure 5. Polymerase chain amplification analysis of the c-sis gene in members of the family predisposed to meningioma and in sporadic meningiomas. PCR products analyzed by agarose gel electrophoresis are shown using DNA samples from (a) lymphocytes of members of family predisposed to meningiomas and $(b)$ brain tumor samples. The brain tumor samples included meningiomas (samples 1, 3, 5, 6, 7, 11, 12, 14, and 15), an oligodendroglioma (sample 2), a metastatic adenocarcinoma (sample 4), gliomas (samples 8 and 13), and a metastatic malignant melanoma (sample 9). Also shown are PCR products from the HOS or H9 lymphoid cell line (also labeled sample 10), a normal blood donor L.R., the M.D. lymphoid cell line obtained from patient II.8, and the bacteriophage clone of the deleted allele from patient II.8. To the left of each photograph is the size of the marker $(M)$, DNA fragments in kilobases.

The structural alteration of the c-sis gene noted in leukocyte DNA, from both of the family members with meningioma who were available for analysis and the one additional family member without meningioma (12), was not found in six additional family members or 25 unrelated and unaffected individuals (Fig. 1; data not shown). Thus, this abnormality may be closely associated with meningioma development in this family. 
Analysis of the structural abnormality using several different restriction enzyme digestions demonstrated DNA fragments $0.1 \mathrm{~kb}$ smaller than those in DNA from unaffected individuals (Fig. 2). Thus, the abnormality in the c-sis gene represents a deletion rather than a novel Pst I site.

The location of the deletion within an intron (Fig. 3) and an Alu sequence (Fig. 4) contributes to a growing number of observations that these sequences are not without some function $(27,28)$. For example, the presence of a 15-nucleotide flanking sequence suggests that Alu sequences may be involved in transposition. Alternatively, the deletion of precisely one copy of the 130-bp repeat sequence and the intervening 5-bp sequence (Fig. 4) suggests that this deletion arose by homologous recombination between identical sequences.

Is the c-sis deletion associated with meningioma development? The finding of an identical deletion in both the familial cases and a sporadic case of meningioma suggests an association exists. Numerous samples from other meningioma patients and unaffected individuals are being examined to assess whether there is a statistically significant association of this genetic abnormality with sporadic meningiomas. Additional information about the role of this gene in oncogenesis depends on analysis of c-sis genetic alterations in individuals with other central nervous systems tumors, or individuals with conditions such as neurofibromatosis which predispose to meningiomas and other central nervous system tumors.

How might the c-sis deletion be related to meningioma development? One hypothesis is that a recessive oncogene, either c-sis or a gene in close proximity to c-sis on chromosome 22 , may be involved in meningioma development. If c-sis has this activity, the deletion in the fifth intron may inactivate one allele, and the other allele may be inactivated in the tumor. This would be analogous to the function of suppressor genes involved in the development of retinoblastoma, osteosarcoma, and Wilm's tumor $(29,30)$. An alternate explanation is that the deletion in the fifth intron activates the expression of the c-sis gene. A point mutation in the fourth intron of c-Ha-ras is also associated with overexpression (27). Since some Alu sequences act as RNA polymerase III promoters (28), this activity may disrupt expression of the c-sis gene. Analysis of c-sis expression from the deleted allele and normal alleles is in progress to test these hypotheses.

\section{Acknowledgments}

We thank T. Philpott and B. Trowbridge for technical assistance; $P$ Lowensteine and M. Green (St. Louis University), G. Brodeur (Washington University), and Sandra Bigner (Duke University) for brain tumor samples; B. Downton for DNA from neurofibromatosis type 1 patients; L. Westfield and E. Sadler (Howard Hughes Medical Institute), and G. Grant (Washington University Protein Chemistry Facility) for the oligonucleotides; and G. Brodeur, T. Deuel, and S. Korsmeyer for critical review of the manuscript.

This work was supported by the Washington University BRS Committee, the American Cancer Society, and the Washington University-Monsanto Research Agreement. Dr. Ratner is a Hartford Foundation fellow.

\section{References}

1. Dalla Favera, R., R. C. Gallo, A. Giallongo, and C. M. Croce. 1982. Chromosomal localization of the human homolog (c-sis) of the simian sarcoma virus onc gene. Science (Wash. DC). 218:686-688.

2. Josephs, S. F., G. Chan, L. Ratner, and F. Wong-Staal. 1985. Human protooncogene nucleotide sequences corresponding to the transforming region of simian sarcoma virus. Science (Wash. DC). 223:487-491.

3. Collins, T., D. Ginsburg, J. M. Boss, S. H. Orkin, and J. S. Pober. 1985. Cultured human endothelial cells express platelet-derived growth factor B chain: cDNA cloning and structural analysis. Nature (Lond.). 316:748-750.

4. Martinet, Y., P. B. Bitterman, J.-F. Morenx, G. R. Grotendorst, G. R. Martin, and R. G. Crystal. 1986. Activated human monocytes express the $c$-sis protooncogene and release a mediator showing PDGF-like activity. Nature (Lond.). 319:158-160.

5. Seifert, R. A., S. M. Schwartz, and D. F. Bowen-Pope. 1984. Developmentally regulated production of platelet-derived growth factor-like molecules. Nature (Lond.). 311:669-671.

6. Richardson, W. D., N. Pringle, M. J. Mosley, B. Westermark, and M. Dubois-Dalcq. 1988. A role for platelet-derived growth factor in normal gliogenesis in the central nervous system. Cell. 53:309-319.

7. Westin, E. H., F. Wong-Staal, E. P. Gelmann, R. Dalla Favera, T. S. Papas, J. A. Lautenberger, A. Eva, E. P. Reddy, S. R. Tronick, S. A. Aaronson, and R. C. Gallo. 1982. Expression of cellular homologues of retroviral onc genes in human hematopoietic cells. Proc. Natl. Acad. Sci. USA. 79:2490-2494.

8. Robbins, K. C., S. G. Devare, E. P. Reddy, and S. A. Aaronson. 1982. In vivo identification of the transforming gene product of simian sarcoma virus. Science (Wash. DC). 218:1131-1133.

9. Wolfe, L. G., and F. Deinhardt. 1978. Overview of viral oncology studies in Saguinus and Callithrix species. Primates Med. 10:96118.

10. Zang, K. D. 1982. Cytological and cytogenetical studies of human meningioma. Cancer Genet. Cytogenet. 6:249-274.

11. Seizinger, B. R., S. De la Monte, L. Atkins, J. F. Gusella, and R. L. Martuza. 1987. Molecular genetic approach to human meningioma: loss of genes of chromosome 22. Proc. Natl. Acad. Sci. USA. 84:5419-5423.

12. Bolger, G. B., J. Stamberg, I. R. Kirsch, G. F. Hollis, D. F. Schwarz, and G. H. Thomas. 1985. Chromosomal translocation $t(14 ; 22)$ and oncogene (c-sis) variant in a pedigree with familial meningioma. N. Engl. J. Med. 312:564-567.

13. Maniatis, T., E. F. Fritsch, and J. Sambrook. 1982. Molecular cloning: a laboratory manual. Cold Spring Harbor Laboratory. Cold Spring Harbor, NY.

14. Ratner, L., B. Thielan, and T. Collins. 1987. Sequences of the 5 portion of the human c-sis gene: characterization of the transcriptional promoter and regulation of expression of the protein product by $5^{\prime}$ untranslated sequences. Nucleic Acids Res. 15:6017-6036.

15. Clarke, M. F., E. Westin, D. Schmidt, S. F. Josephs, L. Ratner, F. Wong-Staal, R. C. Gallo, and M. S. Reitz. 1984. Transformation of NIH 3 T 3 cells by a human $c$-sis cDNA clone. Nature (Lond.). 308:464-467.

16. Maxam, A. M., and W. Gilbert. 1989. Sequencing end-labeled DNA with base-specific chemical cleavages. Methods Enzymol. 65:499-560.

17. Sanger, F., S. Nicklen, and A. R. Coulson. 1977. DNA sequencing with chain-terminating inhibitors. Proc. Natl. Acad. Sci. USA. 74:5463-5467.

18. Feinberg, A. P., and B. Vogelstein. 1983. A technique for radiolabeling DNA restriction endonuclease fragments to high specific activity. Anal. Biochem. 132:6-13.

19. Kariya, Y., K. Kato, Y. Hayashizaki, S. Himeno, S. Tarui, and K. Matsubara. 1987. Revision of consensus sequence of human Alu repeats. A review. Gene (Amst.). 53:1-10.

20. Boldrey, E. 1971. The meningiomas. In Pathology of the Nervous System. J. Minckler, editor. McGraw-Hill Inc., New York. 2125-2144.

21. Rausing, A., W. Ybo, and J. Stenflo. 1970. Intracranial menin- 
gioma. A population study of ten years. Acta Neurol. Scand. 46:102110.

22. Quest, D. O. 1978. Meningiomas: an update. Neurosurgery (Baltimore). 3:219-224.

23. Battersby, R. D. E., J. W. Ironside, and E. L. Maltby. 1986. Inherited multiple meningiomas: a clinical, pathological, and cytogenetic study of an affected family. J. Neurol. Neurosurg. Psychiatry 49:362-368.

24. Delleman, J. W., J. G. Y. De Jong, and G. M. Bleeker. 1978. Meningiomas in five members of a family over two generations, in one member simultaneously with acoustic neurinomas. Neurology. 28:567-570.

25. National Institutes of Health Consensus Development Conference Statement. 1987. Neurofibromatosis. 6:1-7.

26. Therman, E. 1986. Human Chromosomes, Structure, Behav- ior, Effects. Springer-Verlag New York Inc., New York.

27. Cohen, J. B., and A. D. Levinson. 1988. A point mutation in the last intron responsible for increased expression and transforming activity of the c-Ha-ras oncogene. Nature (Lond.). 334:1 19-124.

28. Schmid, C. W., and W. R. Jelinek. 1982. The Alu family of dispersed repetitive sequences. Science (Wash. DC). 216:1065-1070.

29. Hansen, M. F., A. Koufos, B. L. Gallie, R. A. Phillips, O. Fodstad, A. Brogger, T. Gedde-Gahl, and W. K. Cavenee. 1985. Osteosarcoma and retinoblastoma: a shared chromosomal mechanism revealing recessive predisposition. Proc. Natl. Acad. Sci. USA. 82:6216-6220.

30. Van Heyningen, V., P. A. Boyd, A. Seawright, J. M. Fletcher, J. A. Fantes, K. E. Buckton, G. Spowart, D. J. Porteous, R. E. Hill, M. S. Newton, and N. D. Hastie. 1985. Molecular analysis of chromosome 11 deletions in aniridia-Wilms tumor syndrome. Proc. Natl. Acad. Sci. USA. 82:8592-8596. 